\title{
The carboxyl terminus of the Bacillus subtilis SecA is dispensable for protein secretion and viability
}

\author{
Karel H. M. van Wely, ${ }^{1} \dagger$ Jelto Swaving, ${ }^{1}$ Michael Klein, ${ }^{2}$ \\ Roland Freudl ${ }^{2}$ and Arnold J. M. Driessen ${ }^{1}$
}

Author for correspondence: Arnold J. M. Driessen. Tel: +31 50 3632164. Fax: +31 503632154.

e-mail: a.j.m.driessen@biol.rug.nl

\footnotetext{
1 Department of Microbiology, Groningen Biomolecular Sciences and Biotechnology Institute, University of Groningen, Kerklaan 30, $9751 \mathrm{NN}$ Haren, The Netherlands

2 Institut für Biotechnologie 1, Forschungszentrum Jülich $\mathrm{GmbH}$, D-52425 Jülich, Germany
}

\begin{abstract}
The Escherichia coli secretion-dedicated chaperone SecB targets a subset of proteins to the translocase by interacting with the carboxyl (C-) terminus of SecA. This region of SecA is highly conserved in Eubacteria, but despite its presence in the Bacillus subtilis SecA, the B. subtilis genome does not appear to contain a gene for a clear homologue of SecB. Deletion of the C-terminus of the B. subtilis SecA yields cells that have normal viability, but that exhibit a response reminiscent of oxidative stress and the loss of a number of secretory proteins from the culture supernatant. Semi-quantitative RT-PCR demonstrates that these proteins are expressed at lower levels. The C-terminus of SecA fused to glutathione $S$-transferase (GST) specifically binds a cytosolic protein, termed MrgA. This protein has been reported to function in relation to oxidative stress, but deletion of the $m$ rgA gene does not result in a secretion defect nor does it cause an oxidative stress response. It is concluded that the C-terminus of the $B$. subtilis SecA is not essential for secretion and viability.
\end{abstract}

Keywords: protein secretion, chaperones, SecA, SecB

\section{INTRODUCTION}

Bacillus subtilis has become a paradigm for studies on protein secretion in Gram-positive bacteria primarily because bacilli have a high capacity for the production of exoenzymes. Protein secretion across the cytoplasmic membrane of $B$. subtilis is catalysed by a system that is homologous to the precursor protein translocase of Escherichia coli (Overhoff et al., 1991; van Wely et al., 1998) that has been studied in detail. In E. coli, protein translocation is mediated by cytosolic chaperones, the translocation ATPase SecA, and a large integral membrane protein complex with SecY, SecE, SecG, SecD and SecF as subunits (Driessen, 1996). Only SecA, SecE and SecY are essential for viability, and homologues have been identified genetically in B. subtilis. SecA is encoded by the div A gene (Sadaie et al., 1991; Asai et al., 1997), and was originally identified in a set of mutants

†Present address: Department of Experimental Pathology, Josephine Nefkens Institute, Erasmus University Rotterdam, PO Box 1738, 3000 DR Rotterdam, The Netherlands.

Abbreviations: Cam, chloramphenicol; GST, Schistosoma japonicum glutathione $S$-transferase; GST-C, GST fusion bearing the C-terminal 22 amino acids of $B$. subtilis SecA; Phle, phleomycin; SRP, signal recognition particle. conditionally defective in division and sporulation. SecY (Suh et al., 1990; Breitling et al., 1994) and SecE (Jeong et al., 1993) were identified after nucleotide sequence analysis of the chromosomal regions that contain the ribosomal $s p c$ operon and $n u s G$, respectively. The analogous regions in $E$. coli contain sec $Y$ and secE, respectively. Homologues of the SecG (Swaving et al., 1999; van Wely et al., 1999) and SecD/SecF (Bolhuis et al., 1998) proteins have been identified by sequence analysis of the completed B. subtilis chromosome.

Studies in E. coli have demonstrated that prior to their interaction with SecA, precursor proteins may interact with the signal recognition particle (SRP) (Powers \& Walter, 1997; Bunai et al., 1999) or the export-dedicated molecular chaperone SecB (Kumamoto, 1989, 1991). Both pathways converge at the translocase (Valent et al., 1998; Bunai et al., 1999). The bacterial SRP consists of Ffh (Römisch et al., 1989; Honda et al., 1993) and 4.5S RNA (Struck et al., 1989; Powers \& Walter, 1997). In E. coli, SRP interacts with hydrophobic signal sequences of nascent precursor proteins, and targets these nascent chain-ribosome complexes to the membrane through the SRP receptor, FtsY (Luirink et al., 1994; Oguro et al., 1995). The B. subtilis Ffh specifically binds to precursor proteins (Bunai et al., 1996) and promotes 


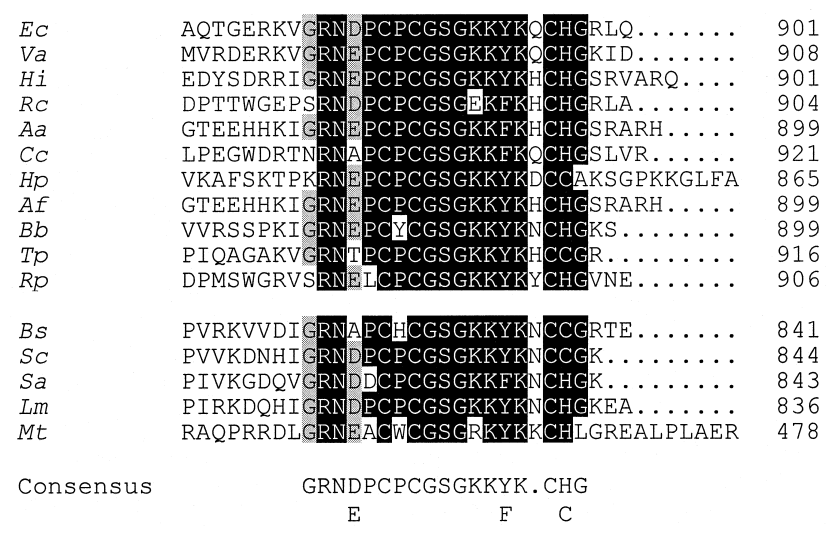

Fig. 1. Multiple sequence alignment of the C-terminal domains of bacterial SecA proteins. Black and grey shading indicate a higher or lesser degree of conservation. Organisms are indicated as follows: Ec, Escherichia coli (PIR:S40402); Va, Vibrio alginolyticus (EMBL:BAA33403); Hi, Haemophilus influenzae (PIR:H64068); Rc, Rhodobacter capsulatus (SWISSPROT:P52966); Aa, Actinobacillus actinomycetemcomitans (EMBL:AAD22450); Cc, Caulobacter crescentus (SWISSPROT:P38380); Hp, Helicobacter pylori (SWISPROT:O25475); Af, Aquiflex aeolicus (TREMBLNEW:G2982840); Bb, Borrelia burgdorferi (EMBL:AAC46347); Tp, Treponema pallidum (SWISS-PROT:Q83394); Rp, Rickettsia prowazekii (SWISSPROT:Q9ZCX7); Bs, Bacillus subtilis (EMBL:E1186051); Sc, Staphylococcus carnosus (SWISS-PROT:P47994); Sa, Staphylococcus aureus (SWISS-PROT:O06446); Lm, Listeria monocytogenes (SWISS-PROT:P47847); Mt, Mycobacterium tuberculosis (EMBL:CAB09972). The conserved region of the $M$. tuberculosis SecA represents an internal sequence.

their binding to SecA (Bunai et al., 1999). Depletion of components of the SRP pathway results in a partial block in protein translocation that seems to vary depending on the precursor protein (Oguro et al., 1996, Hirose et al., 2000).

In E. coli, SecB supports the translocation of a subset of proteins, mainly precursors of outer-membrane proteins (Kumamoto \& Francetiç, 1993). SecB binds to nascent precursor proteins (Randall et al., 1997; Behrmann et al., 1998), and holds them in a translocation-competent conformation (Lecker et al., 1989). SecB subsequently targets these proteins to the SecYEG-bound SecA (Hartl et al., 1990; Fekkes et al., 1997). This targeting event is accomplished by the high-affinity binding of SecB to the carboxyl (C-) terminus of the SecYEG-bound SecA, whereas cytosolic SecA interacts with only poor affinity (Den Blaauwen et al., 1997; Fekkes et al., 1997). The interaction is stimulated by the presence of a precursor protein with a functional signal sequence (Fekkes et al., 1998). During the ATP-dependent initiation of protein translocation, SecB is released from the SecYEG-SecAprecursor complex and recycled to the cytosol (Fekkes et al., 1997).

SecB seems to be present in Gram-negative bacteria only (Fekkes et al., 1998), in particular in Enterobacteriaceae. It is not required for viability of $E$. coli but its gene overlaps with $g p s A$, which encodes a biosynthetic snglycerol-3-phosphate dehydrogenase (Shimizu et al.,
1997). Disruption of the gpsA gene results in a severe growth defect when cells are grown on rich media (Shimizu et al., 1997). On the other hand, the C-terminal SecB-interacting domain of E. coli SecA cannot be disrupted (Breukink et al., 1995). SecA lacking this region still promotes protein translocation but no longer binds SecB nor supports SecB-dependent translocation (Fekkes et al., 1997). Since SecB is obsolete, the observation that cells harbouring SecA with a deleted Cterminus are unable to grow is difficult to understand in terms of an impaired SecB function. SecB appears not to be present in Gram-positive bacteria. Strikingly, the Cterminal SecB binding domain is highly conserved among the bacterial SecA proteins (Fig. 1). In the case of Mycoplasma tuberculosis SecA, the conserved region is found in the middle of the SecA protein sequence. The conserved cysteine and histidine residues present in this domain are involved in the coordination of a zinc ion that stabilizes a tertiary structure of SecA that is needed for the interaction with SecB (Fekkes et al., 1999).

We have investigated the role of the C-terminus of the $B$. subtilis SecA to determine if this region is involved in the binding of a hitherto unidentified homologue or analogue of the E. coli SecB. The data demonstrate that, unlike the situation in E. coli, the C-terminus of the $B$. subtilis SecA protein is not needed for viability and protein translocation. Deletion of this region results in aberrant expression of intracellular and secreted proteins and a response reminiscent of oxidative stress.

\section{METHODS}

Bacterial strains and growth media. Strains were grown in Luria-Bertani broth or agar. When necessary, the medium was supplemented with relevant antibiotics as indicated. Construction of vectors and expression of GST fusion proteins was done with E. coli DH5 $\alpha$ [supE44 AlacU169 ( 880 lacZ $\Delta \mathrm{M} 15)$ hsdR17 recA1 endA1 gyrA96 thi-1 relA1]. All other experiments were done with B. subtilis DB104 [nprE18 aprEA3] (Yang et al., 1984).

Chromosomal disruptions. Deletion of the 22 C-terminal amino acids of SecA (Fig. 1) was accomplished as follows. A gene fragment corresponding to amino acids 670 to 819 , followed by a stop codon, was amplified from chromosomal DNA from strain DB104 as a SacI-XbaI PCR fragment, and cloned into pBluescript SK+. Subsequently, a XbaI-Pst $\mathrm{I}$ fragment containing a phleomycin (Phle) resistance marker and the $1 \cdot 1 \mathrm{~kb}$ Pst I-NcoI chromosomal downstream region were cloned downstream of the partial secA gene, resulting in plasmid pDELC. This vector contains the DB104 chromosomal region with the C-terminal 22 amino acids of the $\sec A$ gene replaced by a Phle resistance marker. Vector pDELC was linearized with PvuII to yield a $2.5 \mathrm{~kb} \sec A \Delta C:$ : phleo fragment and subsequently transformed into B. subtilis DB104 by natural competence (Young, 1967). Phle-resistant colonies resulting from a double crossover were selected. The correct position of the chromosomal replacement was confirmed by PCR. In the resulting strain, DB104 $\Delta$ C, the sec A gene encodes only the first 819 amino acids of the protein.

The $m r g A$ gene of B. subtilis DB104 was disrupted as follows. Regions immediately upstream and downstream of $m \mathrm{rg} A$ were amplified from the chromosome of strain DB104 as BamHI-XbaI and KpnI-HincII cassettes, respectively, and 
cloned into pBluescript SK+. Subsequently, a BglII-PvuIIdigested chloramphenicol (Cam) resistance marker was placed between the BamHI and HincII sites, yielding pDELM. This vector contains the DB104 chromosomal region with the $m r g A$ gene replaced by the Cam resistance marker. Vector pDELM was linearized with PvuII to yield a $2 \cdot 8 \mathrm{~kb}$ fragment containing the $\operatorname{mrg} A:: c a m$ region and subsequently transformed into B. subtilis DB104 by natural competence. Camresistant colonies resulting from a double crossover were selected. The correct position of the chromosomal replacement was confirmed by PCR. In the resulting strain, $\mathrm{DB} 104 \Delta \mathrm{M}$, the Cam resistance gene replaced the $m r g A$ gene while leaving the flanking regions intact. Strain DB104 $\Delta$ CM, carrying both mutations, was generated by transforming

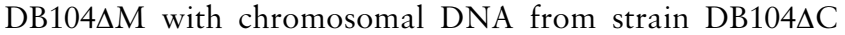
and selecting for Cam and Phle resistance. Since the mutations cause a complete deletion, no selective pressure is needed after the initial selection.

A vector expressing a fusion product of Schistosoma japonicum glutathione $S$-transferase (GST) and the C-terminal 22 amino acids of SecA was generated by ligation of a $102 \mathrm{bp}$ EcoRV-NarI fragment from pMKL4 (Klose et al., 1993) into SmaI-AccI-digested pGEX4T2 (Pharmacia), yielding pET446.

Binding of cytosolic proteins to the C-terminus of SecA. GST and GST-C were purified from cells bearing plasmids pGEX4T2 and pET446, respectively, according to the manufacturer's procedures. After purification, glutathione was removed by dialysis against $50 \mathrm{mM}$ potassium phosphate, $\mathrm{pH} 7 \cdot 0,100 \mathrm{mM} \mathrm{NaCl}$ (buffer A). For the preparation of a cytosolic lysate, B. subtilis cells were broken by French pressure treatment [three times at 8000 p.s.i. $(55 \mathrm{MPa})$ ] and debris was removed by centrifugation at $60000 \mathrm{~g}$ for $45 \mathrm{~min}$. For the binding experiments, GST or GST-C $(5 \mathrm{mg})$ was mixed with the supernatant fraction of the lysate $(100 \mathrm{mg}$ total protein) in buffer $\mathrm{A}$, and incubated for $30 \mathrm{~min}$ on ice. Subsequently, GST or GST-C was reisolated on glutathione Sepharose, washed with 20 column volumes of buffer A, and eluted with one column volume of $25 \mathrm{mM}$ reduced glutathione or alternatively with $50 \mathrm{mM}$ EDTA. The eluted fractions were precipitated with $10 \%$ TCA (final concentration), washed twice with cold acetone and analysed by SDS-PAGE.

Analysis of cellular and secreted proteins. B. subtilis DB104 and its derivatives were grown at $37^{\circ} \mathrm{C}$ in liquid medium. Overnight cultures were diluted 1:50 into fresh medium and grown to the late exponential phase. Cultures were cooled on ice and fractionated into a cellular and a medium fraction by centrifugation. The medium fraction was precipitated with $10 \%$ TCA, washed twice with cold acetone and analysed by SDS-PAGE (van Wely et al., 1999). Cellular pellets were resuspended in sample buffer, sonicated and analysed by SDSPAGE.

Semi-quantitative RT-PCR. Total RNA was isolated from exponentially growing cultures using a total RNA isolation kit (QIAGEN) according to the manufacturer's indications. Firststrand synthesis was accomplished in a single reaction using the access RT-PCR system (Promega) and with primer pairs that specify wapA (forward, 5'-TGTTAAGTCATGGAACTCCGG-3'; backward, 5'-ATCTAATGCCAATTCAGCTCC-3'), ywe A (forward, 5'-TCTTACTTCCTTCGGGCCAAGC-3'; backward, 5'-CCCAAACCGTTTACTACATCGCC-3') or yolA (forward, 5'-TTGCTCTTCTAGCAGTTGTTGC-3' ; backward, 5'-TTTATCCCAGCCTATTACCACC-3'). Subsequently, the relative levels of cDNA were determined in a multiplex PCR in the presence of $\left[{ }^{32} \mathrm{P}\right] \mathrm{dATP}$ with the gap (glyceraldehyde-3-phosphate dehydrogenase) primer pair (forward, 5'-TGCACAACAAACTGCCTTGC-
GC-3'; backward, 5'-TTTACCATGCTGCCTTCCATAAC$\left.3^{\prime}\right)$ alone or in combination with one of the other primer pairs. The PCR products formed after 20 cycles of amplification were separated by electrophoresis on a $6 \%$ denaturing acrylamide gel and quantitated by phosphor-imaging and ImageQuant software (Molecular Dynamics). Values were corrected for gel loading.

Miscellaneous methods. Pulse-chase experiments with $B$. subtilis DB104 and DB104AC expressing E. coli proOmpA or Bacillus licheniformis preAmyL were carried out as described before (Meens et al., 1993). Protein concentrations were determined by the method of Lowry, using BSA as standard. For N-terminal sequencing of polypeptides, samples were separated by SDS-PAGE and blotted onto PVDF. Sequencing was done by the NAPS facility at the University of British Columbia (Vancouver, Canada).

\section{RESULTS}

\section{The C-terminus of SecA is not essential for viability and protein secretion}

The SecB-interacting domain of the E. coli SecA is localized in the C-terminal 22 amino acids (Fekkes et al., 1997; Bunai et al., 1999). This domain is highly conserved among the SecA proteins of Gram-negative and most Gram-positive bacteria, including B. subtilis SecA (Fig. 1). To define the in vivo function of this domain in $B$. subtilis, the last 22 codons of the chromosomal secA gene were replaced by a stop codon. In the resulting strain, DB $104 \Delta \mathrm{C}$, the $\sec A$ gene encodes amino acids 1-819. The truncated SecA protein produced by strain DB104 $\Delta \mathrm{C}$ showed a corresponding higher mobility on SDS-PAGE (Fig. 2). The deletion strain had normal viability and was capable of growing at a rate only slightly slower than the parental strain (data not shown).

To investigate a possible secretion defect, culture supernatants of B. subtilis DB104 and DB104AC were compared (Fig. 3a). In the culture supernatant fraction of strain DB104 $\Delta$ C, a considerable number of proteins appeared to be absent (Fig. 3a, compare lanes 1 and 2). Polypeptides present in the culture supernatant of strain DB104, but absent from that of DB104 $\Delta$ C were blotted and N-terminally sequenced. The resulting sequences QSASIEAK and AEALPLYY correspond to the mature parts of the secretory proteins YweA (molecular mass $16545 \mathrm{Da}$ ) and YolA (17086 Da), respectively. These two proteins co-migrate on SDS-PAGE. Since the yield of the two proteins in the $\mathrm{N}$-terminal sequencing showed

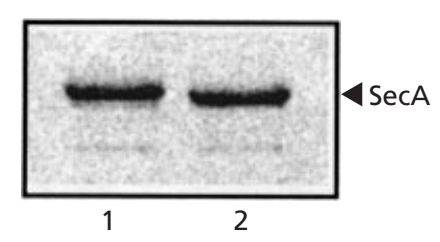

Fig. 2. Western blot analysis of SecA protein from strains DB104 (lane 1) and DB104AC (lane 2). Equal amounts of cellular protein were separated on SDS-PAGE, blotted, and incubated with antibodies directed against $B$. subtilis SecA. 

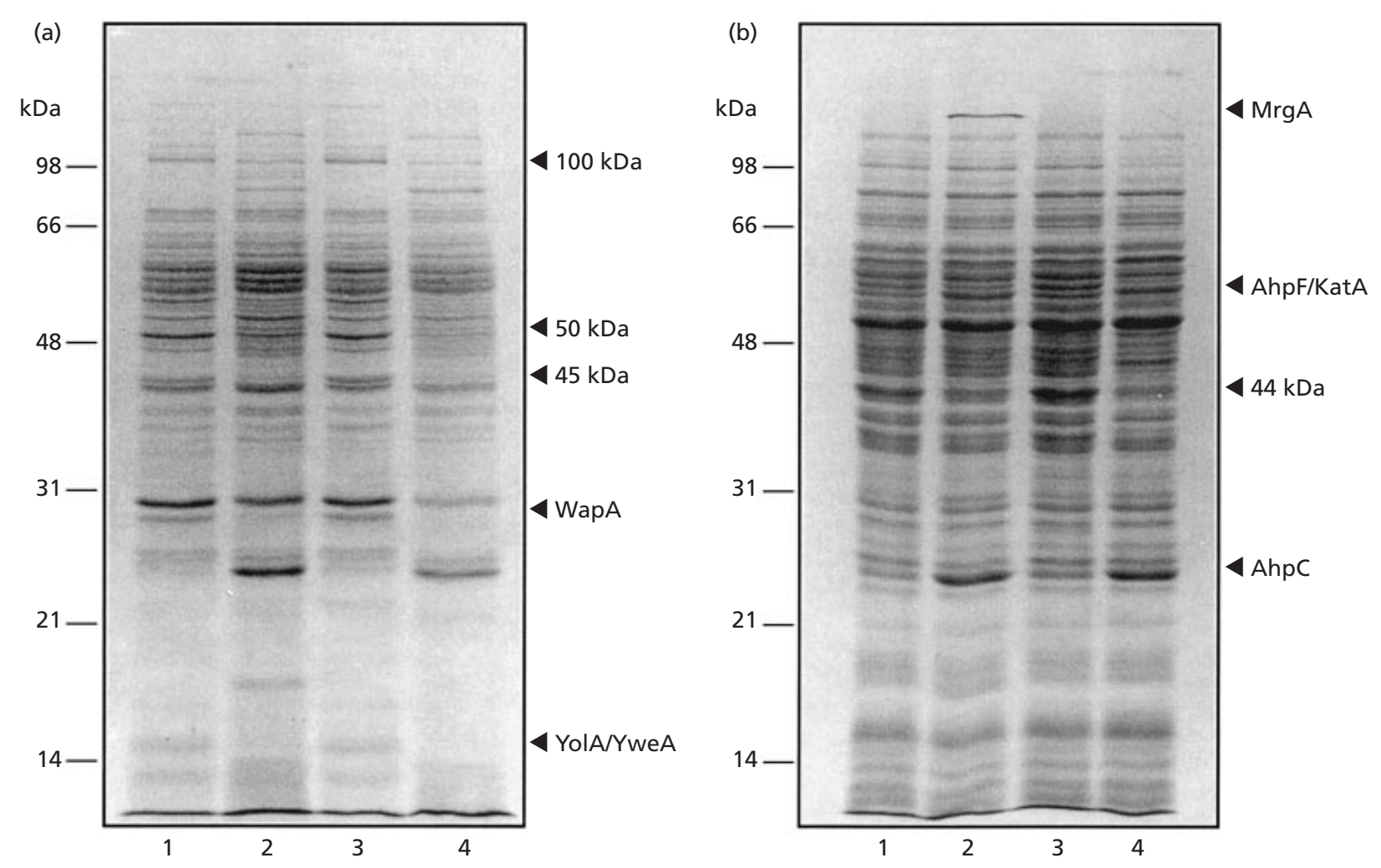

Fig. 3. Coomassie-stained SDS-PAGE of the culture supernatant (a) and cellular (b) fractions of $B$. subtilis strains DB104 (lane 1), DB104 $\Delta C$ (lane 2), DB104 $\Delta M$ (lane 3), and DB104 $\Delta C M$ (lane 4). Cultures were grown to late exponential phase at $37^{\circ} \mathrm{C}$. The position of the molecular mass markers is indicated on the left. The position of AhpC and the tentatively identified AhpF, KatA and MrgA proteins, and of the aberrantly expressed proteins, is indicated on the right.

(a)

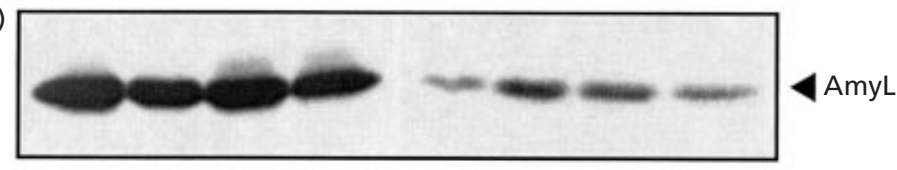

(b)

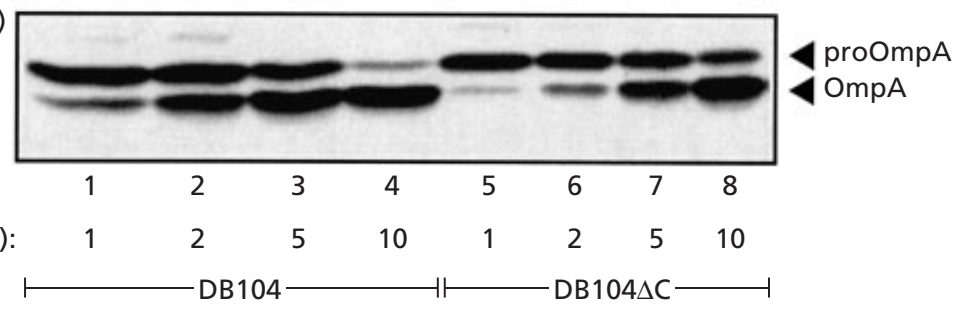

Fig. 4. Pulse-chase experiments of preAmyL (a) and proOmpA (b) in B. subtilis DB104 and DB104AC. Cells were labelled with $\left[{ }^{35}\right.$ S]methionine as described before (Meens et al., 1993), and chased for the indicated times. Positions of AmyL, proOmpA and OmpA are indicated. an equimolar distribution and the polypeptide band has disappeared completely from the mutant, we assume both proteins are affected equally. The $28 \mathrm{kDa}$ protein had a peptide sequence of EVYLDPIH, which corresponds to an internal fragment of WapA (molecular mass $258022 \mathrm{Da}$ ). The $\mathrm{N}$-terminal sequence of the 45, 50 and $100 \mathrm{kDa}$ proteins could not be determined. In addition, some extra bands appeared in the culture supernatant of strain DB104 $\Delta$ C, but these bands correspond to cytosolic polypeptides that are expressed to a high level in this strain (see below), indicating that some lysis has occurred (compare Fig. 3a and 3b).
To examine if the SecA $\Delta \mathrm{C}$ protein supports efficient secretion of precursor proteins, translocation of $B$. licheniformis preAmyL (Fig. 4a) and E. coli proOmpA (Fig. 4b) was analysed in strains DB104 and DB104 $\Delta$ C by pulse-chase experiments. ProOmpA was chosen because this protein requires $\mathrm{SecB}$ for efficient secretion by E. coli. Due to the rapid processing of the precursor, only the mature AmyL protein was detected in either the parental or the mutant strain. Importantly, the mutant strain did not accumulate any precursor, suggesting that translocation is not impaired. With proOmpA, a significant portion of the protein could be detected in the 


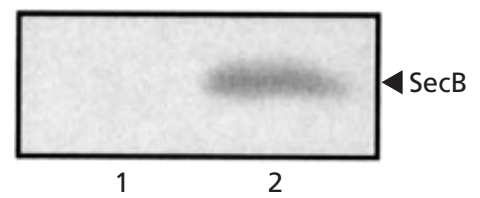

Fig. 5. Binding of the $E$. coli SecB to the C-terminal domain of the $B$. subtilis SecA. The amount of SecB protein associated with (lane 1) GST or (lane 2) the GST-C fusion protein isolated from $E$. coli was determined by Western blot analysis using polyclonal antibodies against SecB.

precursor form after the first chase time and nearly all protein was processed within $10 \mathrm{~min}$. The processing of proOmpA in strain DB104 $\Delta$ C was somewhat slower, but still most of the precursor was processed after 10 min. In both the mutant and the parental strain, translocation of proOmpA was accompanied by proteolysis of the translocated protein into the 16 and $18 \mathrm{kDa}$ fragments (data not shown) (Meens et al., 1997). Taken together these data show that strain DB104 $\Delta$ C is only marginally affected in its secretion abilities, and that the C-terminus of SecA has no function in protein secretion in B. subtilis.

\section{Deletion of the C-terminus of SecA suppresses the expression of wapA and $y w e A$ genes}

Since the pulse-chase experiments did not show striking differences between strains DB104 and DB104 $\Delta$ C, the absence of several protein bands in the supernatant of the DB104 $\Delta$ C cells may not result from a secretion defect but may be caused by an altered expression of the corresponding genes. To obtain an estimate of the relative expression levels of WapA, YweA and YolA in both strains, semi-quantitative RT-PCR was performed. Taking the level of the gap (glyceraldehyde-3-phosphate dehydrogenase) mRNA transcript as 1.0 (the absolute level was identical in DB104 and DB104 $\Delta$ C), the wapA and ywe $A$ transcript levels were 0.45 and 0.89 for DB104, and undetectable and $0 \cdot 27$ for DB104 $\Delta$ C. No significant levels of yolA mRNA could be detected in either strain. These data strongly suggest that a lowering of the expression level accounts for the absence of protein bands in the culture supernatant of strain DB104 $\Delta$ C.

\section{The C-terminus of $B$. subtilis SecA binds $E$. coli SecB and $B$. subtilis MrgA}

When fused to GST, the C-terminal 22 amino acids of the E. coli SecA have been shown to constitute a genuine SecB-binding site (Fekkes et al., 1997). To identify a possible binding partner in B. subtilis, the C-terminal 22 amino acids of B. subtilis SecA were fused to GST to yield GST-C. Both GST and GST-C were expressed in E. coli and purified by glutathione-affinity chromatography. E. coli SecB specifically co-purified with the GST-C fusion protein as detected by Coomassie-stained SDS-PAGE (data not shown) and Western blotting (Fig. 5). The same binding was detected before with the GST fusion protein harbouring the C-terminal 22 amino acids of the E. coli SecA (Fekkes et al., 1997). Binding studies with purified E. coli $\mathrm{SecB}$ confirmed the interaction with the GST-C fusion protein harbouring the $B$. subtilis sequence (data not shown). It is, therefore, concluded that the C-terminus of the B. subtilis SecA provides a valid binding site for $\mathrm{SecB}$.

Next, cytosol of B. subtilis DB104 was incubated with GST and GST-C, and bound polypeptides were analysed by SDS-PAGE. A larger number of proteins associate non-specifically with both GST and GST-C. However, one polypeptide with an apparent molecular mass of $18 \mathrm{kDa}$ was found to specifically copurify with GST-C. Particularly high amounts of this protein bound to GSTC when cytosol derived from strain DB104 $\Delta$ C was used (Fig. 6), although other proteins are expressed to much higher levels in this strain (Fig. 3b). Blotting and subsequent $\mathrm{N}$-terminal amino acid sequencing identified the $18 \mathrm{kDa}$ polypeptide as the $\mathrm{MrgA}$ protein. $\mathrm{MrgA}$ could be eluted from its GST-C bound form by $50 \mathrm{mM}$ EDTA under conditions where GST-C remains attached to the column material.

\section{Deletion of the C-terminus of SecA results in an oxidative stress response}

Since the deletion of the C-terminus of SecA appears to affect protein secretion in an indirect manner, we also compared the cellular polypeptide patterns of B. subtilis DB104 and DB104 $\Delta$ C (Fig. 3b). Striking differences between $\mathrm{DB} 104 \Delta \mathrm{C}$ and its parental strain were also evident in the cellular fractions. Strain DB104 $\Delta$ C showed a dramatic increase in the level of proteins with apparent molecular masses of 25,55 and $120 \mathrm{kDa}$, and a decreased level of a protein with an apparent molecular mass of $44 \mathrm{kDa}$ (Fig. 3b). The $25 \mathrm{kDa}$ protein had an N-terminal sequence of MSLIGKEV, which corresponds to AhpC $(20482 \mathrm{Da})$, a protein involved in oxidative and other forms of stress (Völker et al., 1994; Antelmann et al., 1996). It was not possible to identify the 55 and $120 \mathrm{kDa}$ proteins by $\mathrm{N}$-terminal sequencing. However, they probably correspond to $\mathrm{AhpF}(54705 \mathrm{Da})$ and KatA (54567 Da) running together, and MrgA, respectively. These proteins are expressed together with $\mathrm{AhpC}$ in a coordinated fashion (Chen et al., 1995; Antelmann et al., 1996), and their calculated molecular mass is in perfect agreement with the observed polypeptide bands. The protein band at $44 \mathrm{kDa}$ was not investigated further.

AhpF functions together with AphC in the reduction of alkyl hydroperoxides (Chen et al., 1995), whereas KatA is involved in the reduction of hydrogen peroxide (Bsat et al., 1996). MrgA is synthesized as a $17 \cdot 3 \mathrm{kDa}$ polypeptide, but has been reported to exist in a highmolecular-mass complex that does not completely dissociate even after boiling in SDS (Chen \& Helmann, 1995). The high-molecular-mass form of MrgA confers resistance to oxidative challenge, whereas the form bound by GST-C seems to exclusively represent the monomeric form (Fig. 6). These data indicate that the 
(a)

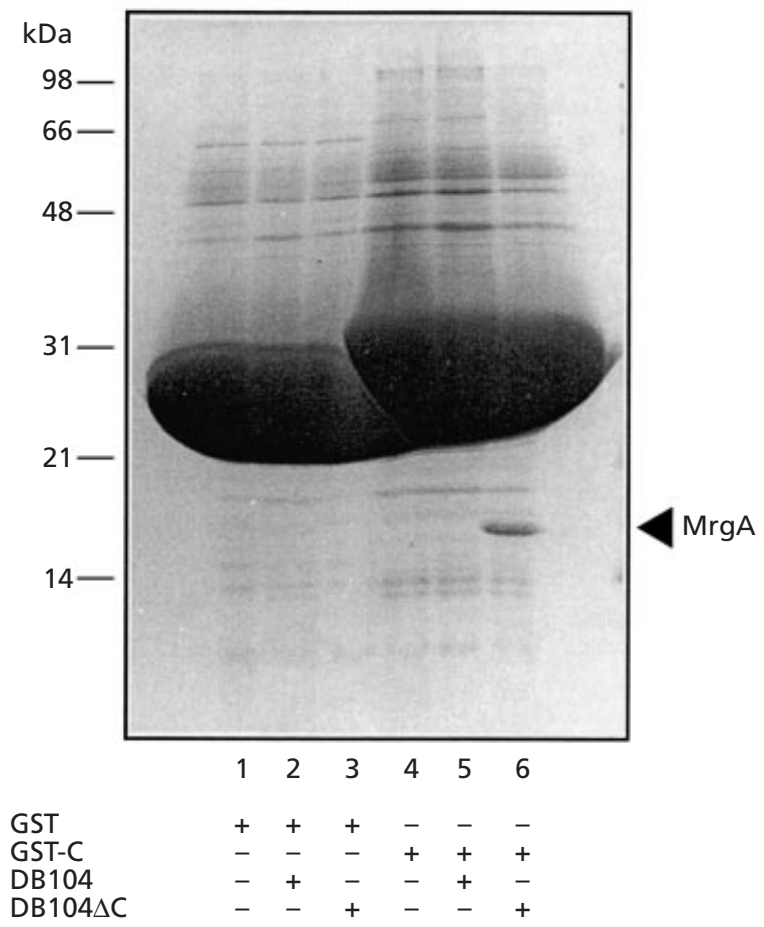

(b)

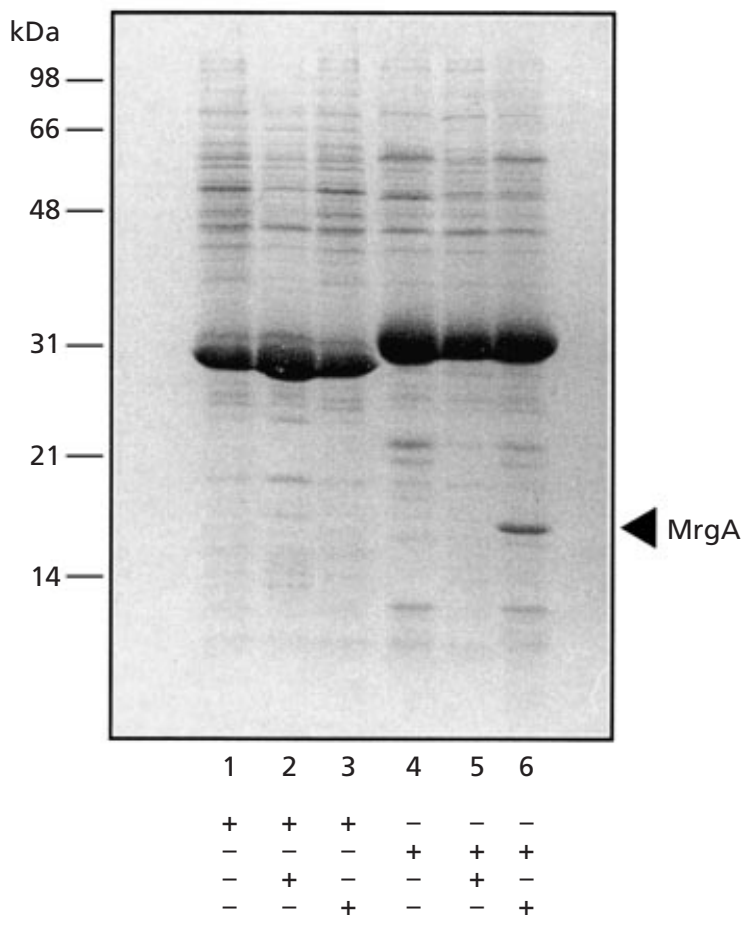

Fig. 6. Specific binding of MrgA to the C-terminus of the B. subtilis SecA. A buffer control (lanes 1 and 4), or a cytosolic lysate of B. subtilis DB104 (lanes 2 and 5) and DB104AC (lanes 3 and 6) was loaded on a glutathione Sepharose column with bound GST (lanes 1-3) or GST-C fusion protein (lanes 4-6), washed, and the bound proteins were eluted with reduced glutathione (a) or EDTA (b). Samples were subsequently analysed on a Coomassie-stained SDS-PAGE gel. The position of the molecular mass markers and the MrgA protein are indicated on the left and right, respectively.

deletion of the C-terminus of SecA elicits a strong oxidative stress response.

\section{MrgA is not involved in protein secretion}

The proposed function of MrgA is to bind to DNA under conditions of oxidative stress and to protect the DNA against damage (Chen \& Helmann, 1995). To gain insight into the possible functional relation between SecA and MrgA, the $\operatorname{mrgA}$ gene was deleted from the chromosome in both DB104 and DB104 $\Delta$ C, yielding

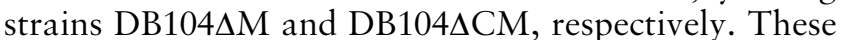
strains had normal viability under the conditions tested. Inactivation of the $m r g A$ gene in the DB104 $\Delta \mathrm{C}$ strain indeed resulted in the loss of the $120 \mathrm{kDa}$ polypeptide, confirming that this protein band corresponds to a highmolecular-mass complex of MrgA (Fig. 3b, lane 4). The DB104 $\triangle \mathrm{CM}$ strain, however, still overproduced the $\mathrm{AhpC}$ and $\mathrm{AhpF}$ proteins to the level observed in strain DB104 $\Delta$ C (Fig. 3b). Comparison of the culture supernatant of the parental strains and $m r g A$ deletion mutants did not reveal any specific differences in the polypeptide pattern except that the overall level of secreted proteins was somewhat reduced (Fig. 3a, lane 4). This demonstrates that MrgA is not directly involved in protein secretion, nor needed for the oxidative stress response induced by the loss of the C-terminus of SecA.

\section{DISCUSSION}

In Enterobacteriaceae, SecB functions as a molecular chaperone dedicated to protein secretion. SecB targets precursor proteins to SecA by binding to a highly conserved sequence in the $\mathrm{C}$-terminus of SecA. With the recent completion of the $B$. subtilis genome sequence (Kunst et al., 1997), it has become clear that a true SecB homologue is not present in this organism. However, the C-terminus of B. subtilis SecA shows a striking homology to the SecB-binding domain of the E. coli SecA (Fig. 1). In this study, we have analysed a possible function of this conserved domain in protein secretion in B. subtilis SecA. Our study shows that in contrast to $E$. coli (Breukink et al., 1995), a chromosomal deletion $B$. subtilis mutant of SecA lacking the putative SecBbinding domain has normal viability and is secretion proficient. Moreover, in vitro binding studies using the C-terminus of the B. subtilis SecA as bait, revealed no cytosolic homologue or analogue of $\mathrm{SecB}$ as binding partner. The C-terminus of the B. subtilis SecA is, however, capable of binding the heterologous E. coli SecB protein, as suggested previously by in vivo studies (Collier, 1994b). In E. coli, translocation of the maltosebinding protein $(\mathrm{MBP})$ strongly depends on SecB. In $B$. subtilis, heterologously expressed preMBP is inefficiently secreted cotranslationally (Collier, 1994a). 
Additional co-expression of SecB indeed caused a shift to post-translational translocation, implying that the $B$. subtilis SecA is capable of binding the heterologous SecB protein. When preMBP was equipped with the signal peptide of $B$. subtilis preAprE, processing of preMBP was no longer stimulated by the co-expression of SecB, and secretion was rather effective (Collier, 1994b). Our experiments show that the export of proOmpA, which in $E$. coli depends on $\mathrm{SecB}$, is only slightly lowered upon deletion of the putative SecB-binding domain of SecA from $B$. subtilis. In conclusion, SecB may influence the secretion of some heterologous precursors when expressed in B. subtilis, but its function seems not to be required for the secretion of homologous precursor proteins.

The screen with the C-terminus of B. subtilis SecA as bait points to the MrgA protein as a cytosolic binding partner. The interaction between the E. coli SecA and SecB is stabilized by a zinc ion (Fekkes et al., 1999). By analogy, chelators could disrupt the observed binding of MrgA to the C-terminus of the B. subtilis SecA. MrgA is not an analogue of SecB, since the deletion of the $\operatorname{mrgA}$ gene from the chromosome did not result in any noticeable secretion defect. The chromosomal replacement of the B. subtilis SecA by a truncate that lacks the putative SecB-binding domain resulted in the loss of specific proteins from the culture supernatant. In combination with the disruption of the $\operatorname{mrg} A$ gene, only a slight overall reduction of secretion was observed, but no additional loss of protein bands from the culture supernatant fraction. Since the specific loss of protein bands could be accounted for by a reduction of expression levels, it appears that the interaction of the C-terminus of SecA with MrgA is not directly related to protein secretion.

The B. subtilis chromosome (Kunst et al., 1997) contains another protein, $\mathrm{YccF}$, that bears at its $\mathrm{N}$-terminus an almost identical sequence as the C-terminus of SecA. The function of YccF is unknown, but its deletion from the chromosome has no notable effect on the growth of B. subtilis (J. M. van Dijl, personal communication). The conserved sequence present in $\mathrm{YccF}$ may also represent a zinc-binding site that is involved in the binding of another protein or even MrgA. Since YccF has not been implicated in protein translocation, we have not analysed this possibility further.

A striking observation is the highly elevated expression of the cytosolic protein AhpC (and tentatively the AhpF and KatA proteins) in the SecA $\Delta \mathrm{C}$ strain. AhpC and AhpF mediate the enzymic reduction of various alkyl hydroperoxides, whereas KatA mainly reduces hydrogen peroxide (Bsat et al., 1996). MrgA confers resistance to oxidative stress by the protection of DNA when present in a high-molecular-mass complex that is stable in SDS-PAGE (Chen \& Helmann, 1995). Together, $a h p C F$, kat $A$ and $m r g A$ form the peroxide regulon. The peroxide regulon is normally induced during oxidative stress or metal limitation (Völker et al., 1994; Chen et al., 1995; Bsat et al., 1996), although AhpC is induced under various other conditions (Völker et al., 1994; Antelmann et al., 1996). Even though oxidative stress is the strongest known inducer of the peroxide regulon, the expression of $a h p C F, \operatorname{mrg} A$ and $k a t A$ is under control of a complex regulatory regime and is influenced by other factors. Expression of these proteins is growthphase dependent, i.e. induced upon entry into stationary phase (Bol \& Yasbin, 1994; Chen et al., 1995). Mutation of AhpC leads to an increased expression of the other proteins in the regulon (Antelmann et al., 1996; Bsat et al., 1996). Finally, the growth-phase-dependent but not the peroxide-induced expression of KatA is abolished by mutation of spoOA (Bol \& Yasbin, 1994). The exact mechanism by which deletion of the C-terminus of SecA induces expression of the peroxide regulon is unknown. The same mutation however results in the loss of a number of distinct polypeptides in the culture supernatant. One of these proteins is WapA, which is secreted as a very large (2334 amino acids) precursor that is subsequently processed into a number of smaller polypeptides with various functions (Foster, 1993). The loss of these proteins from the supernatant of strain DB104 $\Delta$ C probably results from a regulatory process. Synthesis of preWapA is controlled by the DegS-DegU system (Dartois et al., 1998) and affected by salt stress like that of many other secretory proteins (Kunst \& Rapoport, 1995). WapA, as well as YweA and YolA, may also be repressed by the stress response that is evoked in strain DB104 $\Delta$ C. Mutations in the $\sec A$ gene of $B$. subtilis have been shown to affect transcription from spoOA-dependent promoters (Asai et al., 1997, 1998), and thus probably affect transcription of the peroxide regulon (Bol \& Yasbin, 1994). In the case of SecA $\Delta C$, a similar common mechanism could cause the stress response and the repression of a set of secretory proteins.

The question arises whether there is a need for molecular chaperones such as $\operatorname{SecB}$ in protein secretion in $B$. subtilis. Although this question cannot be answered at this time, there are a number of notable differences between E. coli and B. subtilis. Most of the secretory proteins that interact with $\mathrm{SecB}$ in E. coli are outermembrane proteins (Kumamoto \& Francetiç, 1993). Due to their hydrophobic nature, these proteins tend to aggregate when not stabilized by chaperones in solution. $B$. subtilis completely lacks such proteins, and mainly produces soluble exoenzymes that fold rapidly only outside the cell when in contact with calcium (Leloup et al., 1997). A recent report indicates that most exported proteins in $B$. subtilis require $\mathrm{Ffh}$ for secretion (Hirose et al., 2000). The B. subtilis Ffh has been shown to bind specifically to precursor proteins and stimulates their association with the soluble form of SecA (Bunai et al., 1996, 1999). This has led to the suggestion that Ffh fulfils a general chaperone role in the export of secretory protein in B. subtilis, unlike the E. coli Ffh that is primarily needed for the targeting of nascent membrane proteins to the translocase (Valent et al., 1997, 1998).

In conclusion, our data suggest that the conserved Cterminal domain of B. subtilis SecA is not essential for 
protein secretion and viability, and indicate a regulatory function rather than a role in chaperone binding.

\section{ACKNOWLEDGEMENTS}

These investigations were supported by CEC Biotech grants BIO2 CT 930254 and BIO4 CT 960097. The authors thank Dr A. M. Picon for stimulating discussions.

\section{REFERENCES}

Asai, K., Kawamura, F., Sadaie, Y. \& Takahashi, H. (1997). Isolation and characterization of a sporulation initiation mutation in the Bacillus subtilis secA gene. J Bacteriol 179, 544-547.

Asai, K., Fujita, M., Kawamura, F., Takahashi, H., Kobayashi, Y. \& Sadaie, Y. (1998). Restricted transcription from sigma $\mathrm{H}$ or phosphorylated spoOA dependent promoters in the temperature sensitive secA341 mutant of Bacillus subtilis. Biosci Biotechnol Biochem 62, 1707-1713.

Antelmann, H., Engelmann, S., Schmid, R. \& Hecker, M. (1996). General and oxidative stress responses in Bacillus subtilis: cloning, expression, and mutation of the alkyl hydroperoxide reductase operon. J Bacteriol 178, 6571-6578.

Behrmann, M., Koch, H.-G., Hengelage, T., Wieseler, B., Hoffschulte, H. K. \& Müller, M. (1998). Requirements for the translocation of elongation-arrested, ribosome-associated OmpA across the plasma membrane of Escherichia coli. J Biol Chem 273, 13898-13904.

Bol, D. K. \& Yasbin, R. E. (1994). Analysis of the dual regulatory mechanisms controlling expression of the vegetative catalase gene of Bacillus subtilis. J Bacteriol 176, 6744-6748.

Bolhuis, A., Broekhuizen, C. P., Sorokin, A., van Roosmalen, M., Venema, G., Bron, S., Quax, W. J. \& van Dijl, J. M. (1998). SecDF of Bacillus subtilis, a molecular siamese twin required for the efficient secretion of proteins. J Biol Chem 274, 21217-21224.

Breitling, R., Schlott, B. \& Behnke, D. (1994). Modulation of the spc operon affects growth and protein secretion in Bacillus subtilis. J Basic Microbiol 34, 145-155.

Breukink, E., Nouwens, N., van Raalte, A., Mizushima, S., Tommassen, J. \& de Kruijff, B. (1995). The carboxyterminus of SecA is involved in both lipid binding and SecB binding. J Biol Chem 270, 7902-7907.

Bsat, N., Chen, L. \& Helmann, J. D. (1996). Mutation of the Bacillus subtilis alkyl hydroperoxide reductase $(a h p C F)$ operon reveals compensatory interactions among hydrogen peroxide stress genes. J Bacteriol 178, 6579-6586.

Bunai, K., Takamatsu, H., Orinaka, T., Oguro, A., Nakamura, K. \& Yamane, K. (1996). Bacillus subtilis Ffh, a homologue of mammalian SRP, can intrinsically bind to the precursors of secretory proteins. Biochem Biophys Res Commun 227, 762-767.

Bunai, K., Yamada, K., Hayashi, K., Nakamura, K. \& Yamane, K. (1999). Enhancing effect of Bacillus subtilis Ffh, a homologue of the SRP54 subunit of the mammalian signal recognition particle, on the binding of SecA to precursors of secretory proteins in vitro. J Biochem 125, 151-159.

Chen, L. \& Helmann, J. D. (1995). Bacillus subtilis MrgA is a $\operatorname{Dps}($ PexB) homologue: evidence for metalloregulation of an oxidative stress gene. Mol Microbiol 18, 295-300.

Chen, L., Keramati, L. \& Helmann, J. D. (1995). Coordinate regulation of Bacillus subtilis stress genes by hydrogen peroxide and metal ions. Proc Natl Acad Sci U S A 92, 8190-8194.

Collier, D. N. (1994a). Escherichia coli signal peptides direct inefficient secretion of an outer membrane protein (OmpA) and periplasmic proteins (maltose-binding protein, ribose-binding proteins, and alkaline phosphatase) in Bacillus subtilis. J Bacteriol 176, 3013-3020.

Collier, D. N. (1994b). Expression of Escherichia coli SecB in Bacillus subtilis facilitates secretion of the SecB-dependent maltose-binding protein of E. coli. J Bacteriol 176, 4937-4940.

Dartois, V., Débarbouillé, M., Kunst, F. \& Rapoport, G. (1998). Characterization of a novel member of the DegS-DegU affected by salt stress in Bacillus subtilis. J Bacteriol 180, 1855-1861.

Den Blaauwen, T., Terpetschnig, E., Lakowicz, J. R. \& Driessen, A. J. M. (1997). Interaction of SecB with soluble SecA. FEBS Lett 416, 35-38.

Driessen, A. J. M. (1996). Translocation of proteins across the bacterial cytoplasmic membrane. In Handbook of Biophysics, vol. 2, Transport Processes in Membranes, pp. 759-790. Edited by W. N. Konings, H. R. Kaback \& J. S. Lolkema. Amsterdam: Elsevier.

Fekkes, P., van der Does, C. \& Driessen, A. J. M. (1997). The molecular chaperone $\mathrm{SecB}$ is released from the carboxy-terminus of SecA during initiation of precursor protein translocation. EMBO J 16, 6105-6113.

Fekkes, P., de Wit, J. G., van der Wolk, J. P. W., Kimsey, H. H., Kumamoto, C. A. \& Driessen, A. J. M. (1998). Preprotein transfer to the Escherichia coli translocase requires the cooperative binding of SecB and the signal sequence to SecA. Mol Microbiol 29, 1179-1190.

Fekkes, P., de Wit, J. G., Boorsma, A., Friesen, R. H. \& Driessen, A. J. M. (1999). Zinc stabilizes the SecB binding site of SecA. Biochemistry 38, 5111-5116.

Foster, S. J. (1993). Molecular analysis of three major wallassociated proteins from Bacillus subtilis 168: evidence for processing of the product of a gene encoding a $258 \mathrm{kDa}$ precursor two-domain ligand-binding protein. Mol Microbiol 8, 299-310.

Hartl, F.-U., Lecker, S., Schiebel, E., Hendrick, J. P. \& Wickner, W. (1990). The binding of SecB to SecA to SecY/E mediates preprotein targeting to the Escherichia coli membrane. Cell 63, 269-279.

Hirose, I., Sano, K., Shioda, I., Kumano, M., Nakamura, K. \& Yamane, K. (2000). Proteome analysis of Bacillus subtilis extracellular proteins: a two-dimensional protein electrophoretic study. Microbiology 146, 65-75.

Honda, K., Nakamura, K., Nishiguchi, M. \& Yamane, K. (1993). Cloning and characterisation of a Bacillus subtilis gene encoding a homolog of the 54-kilodalton subunit of mammalian signal recognition particle and Escherichia coli Ffh. J Bacteriol 175, 4885-4894.

Jeong, S. M., Yoshikawa, H. \& Takahashi, H. (1993). Isolation and characterization of the secE homologue gene of Bacillus subtilis. Mol Microbiol 10, 133-142.

Klose, M., Schimz, K.-L., van der Wolk, J., Driessen, A. J. M. \& Freudl, R. (1993). Lysine 106 of the Bacillus subtilis SecA protein is required for functional complementation in Escherichia coli secA mutants in vivo. J Biol Chem 268, 4504-4510.

Kumamoto, C. A. (1989). Escherichia coli SecB protein associates with exported protein precursors in vivo. Proc Natl Acad Sci U S A 86, 5320-5324.

Kumamoto, C. A. (1991). Molecular chaperones and protein translocation across the Escherichia coli inner membrane. Mol Microbiol 5, 19-22. 
Kumamoto, C. A. \& Francetiç, O. (1993). Highly selective binding by an Escherichia coli chaperone protein in vivo. J Bacteriol 175, 2184-2188.

Kunst, F. \& Rapoport, G. (1995). Salt stress is an environmental signal affecting degradative enzyme synthesis in Bacillus subtilis. J Bacteriol 177, 2403-2407.

Kunst, F., Ogasawara, N., Moszer, I. \& 148 other authors (1997). The complete genome sequence of the Gram-positive bacterium Bacillus subtilis. Nature 390, 249-256.

Lecker, S., Lill, R., Ziegelhoffer, T., Georgopoulos, C., Bassford, P. J., Kumamoto, C. A. \& Wickner, W. (1989). Three pure chaperone proteins of Escherichia coli-SecB, trigger factor and GroEL - form soluble complexes with precursor proteins in vitro. EMBO J 8, 2703-2709.

Leloup, L., Haddaoui, E.-A., Chambert, R. \& Petit-Glatron, M. F. (1997). Characterization of the rate-limiting step of the secretion of Bacillus subtilis $\alpha$-amylase overproduced during the exponential phase of growth. Microbiology 143, 3295-3303.

Luirink, J., Hagen-Jongman, C. M., van der Weijden, C. C., Oudega, B., High, S., Dobberstein, B. \& Kusters, R. (1994). An alternative protein targeting pathway in Escherichia coli: studies on the role of FtsY. EMBO J 13, 2289-2296.

Meens, J., Frings, E., Klose, M. \& Freudl, R. (1993). An outer membrane protein (OmpA) of Escherichia coli can be secreted across the cytoplasmic membrane of Bacillus subtilis. Mol Microbiol 9, 847-855.

Meens, J., Herbort, M., Klein, M. \& Freudl, R. (1997). Use of the pre-pro part of Staphylococcus byicus lipase as a carrier for secretion of Escherichia coli outer membrane protein A (OmpA) prevents proteolytic degradation of OmpA by cell-associated protease(s) in two different gram-positive bacteria. Appl Environ Microbiol 63, 2814-2820.

Oguro, A., Kakeshita, H., Honda, K., Takamatsu, H., Nakamura, K. \& Yamane, K. (1995). srb: a Bacillus subtilis gene encoding a homologue of the alpha-subunit of the mammalian signal recognition particle receptor. DNA Res 2, 95-100.

Oguro, A., Kakeshita, H., Takamatsu, H., Nkamura, K. \& Yamane, K. (1996). The effect of Srb, a homologue of the mammalian SRP receptor alpha-subunit, on Bacillus subtilis growth and protein translocation. Gene 172, 17-24.

Overhoff, B., Klein, M., Spies, M. \& Freudl, R. (1991). Identification of a gene fragment which codes for the 364 amino-terminal amino acid residues of a SecA homologue from Bacillus subtilis: further evidence for the conservation of the protein export apparatus in gram-positive and gram-negative bacteria. Mol Gen Genet 228, 417-423.

Powers, T. \& Walter, P. (1997). Co-translational protein targeting catalyzed by the Escherichia coli signal recognition particle and its receptor. EMBO J 16, 4880-4886.

Randall, L. L., Topping, T. B., Hardy, S. J. S., Pavlov, M. Y., Freistroffer, D. V. \& Ehrenberg, M. (1997). Binding of SecB to ribosome-bound polypeptide has the same characteristics as binding to full-length denatured proteins. Proc Natl Acad Sci U S A 94, 802-807.

Römisch, K., Webb, J., Herz, J., Prehn, S., Frank, R., Vingron, M. \& Dobberstein, B. (1989). Homology of $54 \mathrm{~K}$ protein of signal recognition particle, docking protein and two E. coli proteins with putative GTP-binding domains. Nature 340, 478-482.

Sadaie, Y., Takamatsu, H., Nakamura, K. \& Yamane, K. (1991). Sequencing reveals similarity of the wild-type div $^{+}$gene of Bacillus subtilis to the Escherichia coli secA gene. Gene 98, 101-105.

Shimizu, H., Nishiyama, K. \& Tokuda, H. (1997). Expression of gpsA encoding biosynthetic sn-glycerol 3-phosphate dehydrogenase suppresses both the $\mathrm{LB}^{-}$phenotype of a $\sec B$ null mutant and the cold-sensitive phenotype of a $\sec G$ null mutant. Mol Microbiol 26, 1013-1021.

Struck, J. C. R., Hartmann, R. K., Toschka, H. Y. \& Erdmann, V. A. (1989). Transcription and processing of Bacillis subtilis small cytoplasmic RNA. Mol Gen Genet 215, 478-482.

Suh, J. W., Boylan, S. A., Thomas, S. M., Dolan, K. M., Oliver, D. B. \& Price, C. W. (1990). Isolation of a $\sec Y$ homologue from Bacillus subtilis: evidence for a common protein export pathway in eubacteria. Mol Microbiol 4, 305-314.

Swaving, J., van Wely, K. H. M. \& Driessen, A. J. M. (1999). Host specific functions of preprotein translocase subunits. J Bacteriol 181, 7021-7027.

Valent, Q. A., de Gier, J.-W. L., von Heijne, G., Kendall, D. A., ten Hagen-Jongeman, C. M., Oudega, B. \& Luirink, J. (1997). Nascent membrane and presecretory proteins synthesized in Escherichia coli associate with signal recognition particle and trigger factor. Mol Microbiol 25, 53-64.

Valent, Q. A., Scotti, P. A., High, S., de Gier, J.-W. L., von Heijne, G., Lentzen, G., Wintermeyer, W., Oudega, B. \& Luirink, J. (1998). The Escherichia coli SRP and Sec targeting pathways converge at the translocon. EMBO J 17, 2504-2512.

Völker, U., Engelmann, S., Maul, B., Riethdorf, S., Völker, A., Schmid, R., Mach, H. \& Hecker, M. (1994). Analysis of the induction of general stress proteins of Bacillus subtilis. Microbiology 140, 741-752.

van Wely, K. H. M., Swaving, J. \& Driessen, A. J. M. (1998). Translocation of the precursor of $\alpha$-amylase into Bacillus subtilis membrane vesicles. Eur J Biochem 255, 690-697.

van Wely, K. H. M., Swaving, J., Broekhuizen, C. P., Rose, M., Quax, W. J. \& Driessen, A. J. M. (1999). Functional identification of the product of the Bacillus subtilis yvaL gene as a SecG homologue. J Bacteriol 181, 1786-1792.

Yang, M. Y., Ferrari, E. \& Henner, D. J. (1984). Cloning of the neutral protease gene of Bacillus subtilis and the use of the cloned gene to create an in vitro-derived deletion mutation. J Bacteriol 160, 15-21.

Young, F. E. (1967). Competence in Bacillus subtilis transformation system. Nature 213, 773-775.

Received 31 March 2000; revised 23 June 2000; accepted 6 July 2000 